\title{
TART 2000: A Coupled Neutron-Photon, 3-D, Combinatorial Geometry, Time Dependent, Monte Carlo Transport Code
}

\author{
D.E. Cullen
}

November 22, 2000

U.S. Department of Energy

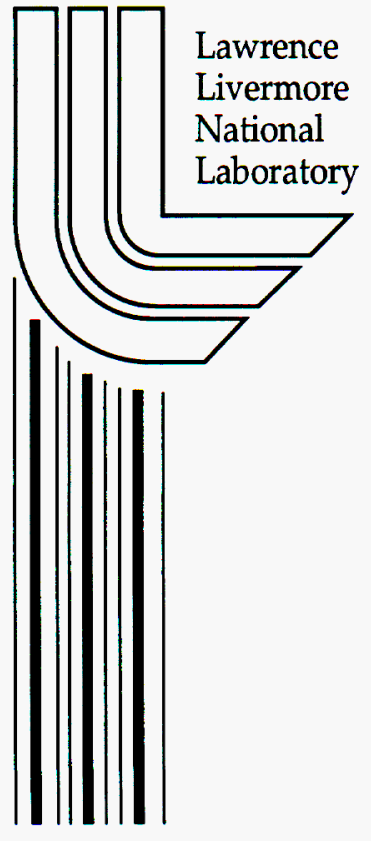





\section{DISCLAIMER}

This document was prepared as an account of work sponsored by an agency of the United States Government. Neither the United States Government nor the University of California nor any of their employees, makes any warranty, express or implied, or assumes any legal liability or responsibility for the accuracy, completeness, or usefulness of any information, apparatus, product, or process disclosed, or represents that its use would not infringe privately owned rights. Reference herein to any specific commercial product, process, or service by trade name, trademark, manufacturer, or otherwise, does not necessarily constitute or imply its endorsement, recommendation, or favoring by the United States Government or the University of California. The views and opinions of authors expressed herein do not necessarily state or reflect those of the United States Government or the University of California, and shall not be used for advertising or product endorsement purposes.

This work was performed under the auspices of the U. S. Department of Energy by the University of California, Lawrence Livermore National Laboratory under Contract No. W-7405-Eng-48.

This report has been reproduced directly from the best available copy.

Available electronically at http://www.doe.gov/bridge

Available for a processing fee to U.S. Department of Energy and its contractors in paper from

U.S. Department of Energy

Office of Scientific and Technical Information

P.O. Box 62

Oak Ridge, TN 37831-0062

Telephone: (865) 576-8401

Facsimile: (865) 576-5728

E-mail: reports@adonis.osti.gov

Available for the sale to the public from

U.S. Department of Commerce

National Technical Information Service 5285 Port Royal Road Springfield, VA 22161

Telephone: (800) 553-6847

Facsimile: (703) 605-6900

E-mail: orders@ntis.fedworld.gov

Online ordering: http://www.ntis.gov/ordering.htm

OR

Lawrence Livermore National Laboratory

Technical Information Department's Digital Library

http://www.llnl.gov/tid/Library.html 



\title{
TART2000 \\ A Coupled Neutron-Photon \\ 3-D, Combinatorial Geometry \\ Time Dependent \\ Monte Carlo Transport Code
}

\author{
by \\ Dermott E. Cullen \\ University of California \\ Lawrence Livermore National Laboratory \\ P.O. Box 808 \\ L-59 \\ Livermore, CA 94550 \\ tele: $925-423-7359$ \\ e. mail: cullen1@llnl.gov
}

November 22, 2000

\begin{abstract}
TART2000 is a coupled neutron-photon, 3 Dimensional, combinatorial geometry, time dependent Monte Carlo radiation transport code. This code can run on any modern computer. It is a complete system to assist you with input preparation, running Monte Carlo calculations, and analysis of output results. TART2000 is also incredibly FAST; if you have used similar codes, you will be amazed at how fast this code is compared to other similar codes. Use of the entire system can save you a great deal of time and energy.

TART2000 is distributed on CD. This CD contains on-line documentation for all codes included in the system, the codes configured to run on a variety of computers, and many example problems that you can use to familiarize yourself with the system.

TART2000 completely supersedes all older versions of TART, and it is strongly recommended that users only use the most recent version of TART2000 and its data files.
\end{abstract}




\section{Dedication}

This report is dedicated to Robert J. Howerton, who for many years led Livermore's Nuclear Data and Monte Carlo efforts. Bob retired in 1986, but continued coming to work until recently, when he finally really retired. Bob was known internationally as well as at the lab for the excellence of his work. Many of our ever changing applications would not have been possible without his efforts to promptly produce nuclear data to meet our needs. Bob will be very much missed both as a co-worker and a friend. We wish Bob all the best for the future, and knowing him as we do, we are sure that he will find other activities to keep him as busy as ever.

\section{Acknowledgments}

I thank the many users of earlier versions of TART who have supplied extremely useful feedback to me. Since the release of TART95, in July 1995, TART96, in November 1996, and TART97 in November 1997, the response from users in terms of feedback has been extremely useful in improving the code. These improvements have been in terms of correcting problems in the initial release of TART95, TART96 and TART97, and in terms of proposing new or improved options to meet the needs of users, now incorporated in TART2000. I highly encourage all users to supply their feedback to me.

\section{The TART2000 System}

This report is intended merely as a brief introduction to TART2000. In particular no graphics results are presented in this report. The on-line documentation for the TART2000 system codes, distributed on TART2000 CD, has been coordinated to illustrate combined use of the codes to make your job simpler and your work easier to accomplish, in particular extensive use of interactive graphics. If you have not used interactive graphics before you are only making your job harder and your tasks will take longer to accomplish.

\section{Overview of This Report}

This report describes all major changes in TART since TART95 [1], including changes in TART96 and TART97. As such this report supersedes the reports of TART96 [2] and TART97 and TART98 [8]. However, the large TART95 report [1] is still the most comprehensive report on TART.

This report is divided into a number of parts, with each part describing one part of the TART2000 CD system. The parts are,

Part 1: TART2000 - Monte Carlo Calculations

Part 2: TARTCHEK - Check TART Input and Display TART Results

Part 3: TARTAID - Create TART Input

Part 4: EPICSHOW - Display Atomic and Nuclear data used by TART

Part 5: PLOTTAB - General Plotting Code to Display TART Output

Part 6: EDITOR - Text editor for use with TART

Part 7: Utility Codes - A collection of Useful Codes

I Strongly Recommend that you read the on-line documentation for all parts of this system, to get a better overall picture of how this entire code system fits together and can help you. The TART2000 online documentation is in Microsoft Word 5.1 format and includes black and white as well as color graphic 
results. Only when you start using the codes in combination will you realize that this is a complete system that can really assist you in your work.

\section{Computer Requirements}

TART2000 will run on any modern Computer, with at Least 8 Megabytes Memory and 30 Megabytes Disk Space. This puppy can run on virtually any computer; see, the below table of running times on a variety of computers.

\section{TART2000 CD}

TART2000 is distributed on CD. This CD contains on-line documentation for all codes included in the system, the codes configured to run on a variety of computers, and many example problems that you can use to familiarize yourself with the system.

\section{TART Home Page}

The TART home page is now located on the web at,

\section{http://reddog1.llnl.gov}

This site contains all of the TART documentation, as well as information and documentation related to TART and the nuclear and atomic data that it uses. This site is periodically updated, with newsletters, etc. If you are a TART user you should periodically check this site for the latest news.

\section{TART Hot Line}

Well, not exactly a hot line, but at least a place to turn to when you need help. If you have any difficulties setting up TART input, running it, or analyzing output, you can contact me at,

\section{Telephone: 925-423-7359}

\section{E. Mail: cullen1@llnl.gov}

\section{Background}

TART2000 is a coupled neutron-photon, 3 Dimensional, combinatorial geometry, time dependent Monte Carlo transport code. The original TARTND has been used and distributed from Lawrence Livermore National Laboratory for many years. TART95, released in July 1995, was the first version of the code designed to be used on virtually any computer. TART96 was designed to extend the general utility of the code to more areas of application, by concentrating on improving the physics used by the code. TART97 further improved the physics, particularly with respect to newer neutron data, and more detailed neutron data. TART2000 further improved the physics, particularly with respect to newer photon data, and more detailed photon data. In additional TART2000 adds new input options, and greatly improved consistency checking designed to make the code more user friendly and to improve the reliability of results. TART 2000 completely supersedes all older versions of TART, and it is strongly recommended that users only use the most recent version of TART2000 and its data files. 


\section{TART95}

The objective of TART95 [1] was to develop a code that is as computer independent as possible. This objective was met by July 1995, when TART95 and its documentation were distributed for general use. At that time TART95 was operational on large mainframe computers, such as CRAY, and workstations, such as: SUN, SGI, HP, DEC Alpha, Meiko, and IBM RISC, as well as IBM-PC. Since that time it has become operational on additional types of computers, such as PowerMAC and even Laptop computers.

TART95 is written in such simple, computer independent FORTRAN, that it can now be easily implemented and used on virtually any computer.

\section{TART96}

Once the objectives of TART95 were met work began on TART96 [2]. The objective of TART96 was to extend the general utility of the code to more areas of application, by concentrating on improving the physics used by the code.

The most important improvements include,

NEW NEUTRON 650 GROUP TREATMENT: for cross sections over the energy range $10^{-4} \mathrm{eV}$ up to $1 \mathrm{GeV}$. Older versions of the code used a 175 group treatment from $1.30910^{-3} \mathrm{eV}$ up to $20 \mathrm{MeV}$, with most of the groups concentrated at higher energy; this limited accurate use of the code to higher energy applications. In contrast the new 650 group treatment is designed to accurately treat the entire neutron energy range, thereby allowing the code to be used for a wider range of applications. As yet neutron data is only generally available up to $20 \mathrm{MeV}$, but as soon as higher energy data becomes available TART96 is ready to use it. If you are a fan of the older 175 group treatment, not be worry: TART96 can use either 175 or 650 groups - the choice is yours.

ENDF/B-VI CROSS SECTIONS: Older versions of the code only used the Livermore ENDL library, which is primarily designed for use in high energy applications. In contrast the ENDF/B-VI data is designed for general use at all energies [3]. Therefore using this data allows the code to be accurately used in a wider range of applications. If you are a fan of the older ENDL data, not be worry: TART96 can use either ENDL or ENDF/B-VI - the choice is yours.

IMPROVED THERMAL SCATTERING TREATMENT: The major advantages of the new thermal scattering treatment include: improved accuracy of sampling, and greatly improved speed of execution [4].

FURTHER IMPROVEMENTS IN COMPUTER INDEPENDENCE: TART95 was implemented on a variety of computers, but it required the use of a few routines that varied from one computer to another. On the basis of user feedback, most of this remaining computer dependence has now been eliminated, and TART96 is now so computer independent that it is almost trivial to implement it on any new type of computer that comes along.

\section{TART97}

Once the objectives of TART96 were met work began on TART97 [8]. The objective of TART97 was to extend the general utility of the code to even more areas of application, by concentrating on improving the 
physics, particularly with regard to improved neutron data, and extending input options used by the code.

The most important improvements include,

NO UPPER LIMITS: on anything you can define by input. Unlike earlier versions of TART, that had a maximum allowed number of zones, surfaces, etc., TART97 has no limits at all. If you want to use a million zones, with a different material composition in each zone, or anything else you can think of, TART97 can handle it.

NO LOWER LIMITS: on anything you can define by input. With earlier versions of TART, that used fixed maximum limits, there was a lot of overhead when running small problems. For example, when TART96 started it used 50 megabytes of memory. TART97 starts with about 1 megabyte and expands to need the needs of each problem. Typical problems only use 3-4 megabytes of memory. I haven't tried it, but TART97 is now so compact it will probably run on the new Palm Top computers.

NEW LONG RUN RANDOM NUMBER GENERATOR: The new generator includes over 2,500 different random number sequences, each sequence a trillion $\left(10^{12}\right)$ random numbers displaced from the preceding sequence. With a modern computer we can generate a trillion random numbers in about one day, if that's all a code is doing. With TART97 each random number sequence should take about 10 to 20 days to use a complete trillion number sequence. Therefore the currently available 2,500 sequences should keep you busy for years; and if you need more, just ask for them.

MULTIPROCESSING: If you have a large computer with say 256 processors and a gigabyte of memory, you can do the arithmetic yourself: for a typical 3-4 megabyte problem, you can run 250 copies of TART97 all at the same time, and use the new utility code TARTSUM to add all of the results together. This approach is completely computer independent, and in this example you can compress 250 days of work into a single day (more than a year of working days into one day). With the new random number generator, using different random number sequences for each run, you can make over 2,500 statistically independent runs and combine the results. You can do this simply by running the same problem with different random number sequences, either using multiprocessing, or any number of single processor computers that you have access to, or a single processor repeatedly, if you just want to run more histories to improve your results.

NEW INPUT OPTIONS: have been added to extend TART97's capabilities, as well as to simplify and make input more user friendly. These options include: cubic and quartic (e.g., torus) surfaces, new rotation and spatial translation, surface cloning, new neutron and photon sources, see, Appendix: Summary of New Conventions and Options.

IMPROVED INPUT CHECKING: to catch more input errors before the calculation begins. As described below, this checking is now incorporated in both TART97 and TARTCHEK. WARNING it is highly recommended that you always use TARTCHEK to check your input, before actually running TART97.

IMPROVED ANALYTICAL VOLUME CALCULATOR: in many cases the results that we are interested in are not results per zone, but rather results per unit volume (e.g. per cc). The improved analytical volume calculator greatly extends TART's ability to quickly calculate zone volumes. This will meet the needs of most applications. For extremely complicated geometric shapes TART97 includes a very fast Monte Carlo volume estimator. Used in combination, the analytical and Monte Carlo volume calculators can quickly define the volume of all of your zones, regardless of how complicated your 
geometry may be.

MODULAR CODING: TART97 is also very modular, so that portions of the code can be used in other codes. For example, TART97 and TARTCHEK use exactly the same input and geometry package; this assures that when you use TARTCHEK to check your geometry, when you run TART97 it will interpret your geometry in exactly the same manner. Similarly these packages will soon be incorporated into EPIC: an Electron Photon Interaction Code.

THE LATEST NEUTRON AND PHOTON DATA: TART97 uses the latest ENDF/B-VI, Release 4, neutron data [5], and Evaluated Photon Data Library '97 (EPDL97), photon data [6]. As with past versions of TART, if you would prefer to use older data, the option is yours.

INTERNAL CONSISTENCY CHECKING: No code is perfect, and for any complicated code, such as TART, that has many possible paths through it, it is virtually impossible to manually check all possible paths. With TART97's new internal consistency checking, it does its own checking every time it is run. For example, every single array in the code is checked for misuse, in an attempt to find as many errors as possible. This procedure has already led to accelerated code development and improvements, and will continue to do so in the future.

GENERAL IMPROVEMENTS: TART97 is based on the older TARTND code, but required massive changes to the code to make it the modern, computer independent code that it is today. As such there were bound to be some growing pains with this essentially new code. Over the last two years, feedback from the many code users has led to general improvements in the code, both in terms of locating and correcting problem areas, as well as in adding and improving code options to meet the needs of users.

FULL OPTIMIZATION: One general improvement worth noting, is that based on communications with a variety of FORTRAN compiler designers, TART97's has been re-designed to allow it to be compiled at the highest level of optimization on most computers, which can greatly reduce running time, without sacrificing accuracy.

TEMPERATURE DEPENDENT NEUTRON DATA: In the past TART has always used nominally room temperature (300 Kelvin) neutron cross sections. We can now prepare additional data files at virtually any temperature to meet programmatic needs [5].

\section{TART98}

Once the objectives of TART97 were met work began on TART98 [8]. The objective of TART98 was to extend the general utility of the code to even more areas of application, by concentrating on improving the physics, particularly with regard to improved photon data, and extending input options used by the code. For details of the new photon treatment see, ref. [9].

It is worthwhile making one overall comment regarding the approach that I have used in implementing TART on every type of computer that I can get my hands on. Initially many people thought this couldn't be done. They were wrong. TART now runs on everything except my wristwatch (I'm working on that). In additional I'1l mention that implementing TART on so many different types of computers has greatly improved the code. Each compiler has strengths and weaknesses, and by testing TART using as many different types of computers as possible has led to locating and eliminating as many different types of potential problems as possible. Isn't using all of these different types of computers time consuming and doesn't it delay development of a code like TART? Not at all. If anything, testing any code on as many 
different computers, using as many different compilers, as possible is probably the fastest way to test a code and improve its reliability.

The most important improvements include,

PHOTON 701 POINT TREATMENT: Incorporated in TART98 is a new photon 701 point treatment for cross sections over the energy range $100 \mathrm{eV}$ up to $1 \mathrm{GeV}$. Older versions of the code used a 176 point treatment from $100 \mathrm{eV}$ up to $30 \mathrm{MeV}$, with most of the points concentrated at higher energy; this limited accurate use of the code to higher energy applications. As with the 650 group neutron treatment, this new treatment of the photon cross sections is designed to accurately treat the entire energy range, allowing the code to be used for a wider range of applications.

PHOTON SCATTERING TREATMENT: TART98 includes an improved treatment of photon coherent and incoherent scattering. The new treatment has the advantage that it is both more accurate, and faster to use, than the older treatment.

MULTIPROCESSING: The TART utility codes MULTIPRO and TARTSUM are now routinely used to perform multiprocessing. This approach to multiprocessing is so simple, straightforward and general that it can be used by virtually all TART users. With this approach if you have a computer with thousands of processors you can use MULTIPRO to create everything that you need to use as many processors as you want and then average the results together using TARTSUM. Even if you don't have a computer with many processors, but you do have access to a number of computers, you can use all available computers to run problems (they don't even have to be the same type of computer), and again use TARTSUM to average all of the results together. This approach is completely computer independent, and in the example case of using 250 processors, you can compress 250 days of work into a single day (more than a year of working days into one day).

IMPROVED NO UPPER OR LOWER LIMITS: on anything you can define by input. Unlike earlier versions of TART, that had a maximum allowed number of zones, surfaces, etc., TART98 has no limits at all. For example, before TART97 the code was limited to a maximum of 1,000 spatial zones. Since then the spatial detail used in TART problems has increased enormously. The largest TART98 problem that I know of involved 27 million $(27,000,000)$ spatial zones. Of course TART98 can still accommodate even the simplest problem, such as a one zone spherical ball. In all cases from smallest to largest TART98 automatically sizes itself to accommodate each individual problem run.

NEW INPUT OPTIONS: have been added to TART98 to allow improved detail in photon tallies. In earlier versions of TART, photon output tallies were always limited to 50 energy bins. Starting with TART98 the user has the option to select 70 (the default), 175, or a full 700 energy bins for photon tallies and output, see, Appendix: Summary of New Conventions and Options.

IMPROVED INPUT CHECKING: to catch more input errors before the calculation begins. As described below, this checking is now incorporated in both TART98 and TARTCHEK. TART98 continues the TART traditions to support all older TART input. For example, if you have a twenty year old TART input problem, you will still be able to use it with TART98. However, TART98 and TARTCHEK are now much more clever at finding errors in TART input, with the result that you may find that TART input decks that ran earlier, will now cause TART to stop, with detailed ERROR messages asking you to correct your input before proceeding.

INTERNAL CONSISTENCY CHECKING: No code is perfect, and for any complicated code, such as 
TART, that has many possible paths through it, it is virtually impossible to manually check all possible paths. Starting with TART97 the code included new internal consistency checking; it did its own checking every time it ran. For example, every single array in the code is checked for misuse, in an attempt to find as many errors as possible. You wouldn't believe how effective this internal checking has been over the last two years at finding and allowing us to eliminate potential problems, resulting in a much more reliable code.

GENERAL IMPROVEMENTS: TART98 is based on the older TARTND code, but required massive changes to the code to make it the modern, computer independent code that it is today. As such there were bound to be some growing pains with this essentially new code. Over the last three years, feedback from the many code users has led to general improvements in the code, both in terms of locating and correcting problem areas, as well as in adding and improving code options to meet the needs of users.

\section{TART2000}

Once the objectives of TART98 were met work began on TART2000. The objective of TART2000 was to extend the general utility of the code to even more areas of application, by concentrating on improving the physics, particularly with regard to improved treatment of neutron fission, and extending input options used by the code

It is worthwhile making one overall comment regarding the approach that $I$ have used in implementing TART on every type of computer that I can get my hands on. Initially many people thought this couldn't be done. They were wrong. TART now runs on everything except my wristwatch (I'm working on that). In additional I'll mention that implementing TART on so many different types of computers has greatly improved the code. Each compiler has strengths and weaknesses, and by testing TART using as many different types of computers as possible has led to locating and eliminating as many different types of potential problems as possible. Isn't using all of these different types of computers time consuming and doesn't it delay development of a code like TART? Not at all. If anything, testing any code on as many different computers, using as many different compilers, as possible is probably the fastest way to test a code and improve its reliability.

The most important improvements include,

THE LATEST NEUTRON INTERACTION DATA: TART2000 includes the latest ENDF/B-VI, Release 7, data. For details of changes in the data, see reference [10]. TART2000 is now distributed only with 650 group ENDF/B-VI neutron interaction data; support of the older 175 group ENDL data has now been dropped.

IMPROVED NEUTRON FISSION TREATMENT: TART2000 includes continuous energy sampling of fission spectra. Earlier versions of TART used equally probable energy bins; equally probable energy bins leads to rather large errors in the low and high energy emission of neutrons. This is a win-win improvement in TART2000, where the new sampling is both more accurate and faster. TART2000 also includes the option of use either the average number of neutrons emitted per fission, or to sample from the distribution of the number of neutrons emitted per fission (sentl 54). Also included in TART2000 is an option to allow spontaneous fission sources (sentl 55). The combination of allowing spontaneous fission sources and sampling from the distribution of the number of neutrons emitted per fission, allows TART2000 to be used to simulate reactor noise analysis problems involving neutron emission correlation effects. 
MULTIPROCESSING: The TART utility codes MULTIPRO and TARTSUM are now routinely used to perform multiprocessing. This approach to multiprocessing is so simple, straightforward and general that it can be used by virtually all TART users. With this approach if you have a computer with thousands of processors you can use MULTIPRO to create everything that you need to use as many processors as you want and then average the results together using TARTSUM. Even if you don't have a computer with many processors, but you do have access to a number of computers, you can use all available computers to run problems (they don't even have to be the same type of computer), and again use TARTSUM to average all of the results together. This approach is completely computer independent, and in the example case of using 250 processors, you can compress 250 days of work into a single day (more than a year of working days into one day). Since the release of MULTIPRO and TARTSUM two years ago, these codes have been significantly improved, based almost exclusively on user feedback, to better meet user needs. This is an excellent example of how the TART system responds to ever changing user needs.

NEW INPUT OPTIONS: have been added to TART2000 to allow reactor noise analysis calculations (sentl 54 and 55), easier simulation of detectors (sentl 56), and definition of zone volumes by user input (volume). For more details, see, Appendix: Summary of New Conventions and Options.

IMPROVED INPUT CHECKING: to catch more input errors before the calculation begins. As described below, this checking is now incorporated in both TART2000 and TARTCHEK. TART2000 continues the TART traditions to support all older TART input. For example, if you have a twenty year old TART input problem, you will still be able to use it with TART2000. However, TART2000 and TARTCHEK are now much more clever at finding errors in TART input, with the result that you may find that TART input decks that ran earlier, will now cause TART to stop, with detailed ERROR messages asking you to correct your input before proceeding.

INTERNAL CONSISTENCY CHECKING: No code is perfect, and for any complicated code, such as TART, that has many possible paths through it, it is virtually impossible to manually check all possible paths. Starting with TART97 the code included new internal consistency checking; it did its own checking every time it ran. For example, every single array in the code is checked for misuse, in an attempt to find as many errors as possible. You wouldn't believe how effective this internal checking has been over the last three years at finding and allowing us to eliminate potential problems, resulting in a much more reliable code. This internal consistency checking has been so effective that since the release of TART98 not one single internal consistency problem has occurred. However, I have left the internal consistency checking in place within the code, so that any changes or new additions to the code are automatically verified. 
GENERAL IMPROVEMENTS: TART2000 is based on the older TARTND code, but required massive changes to the code to make it the modern, computer independent code that it is today. As such there were bound to be some growing pains with this essentially new code. Over the last four years, feedback from the many code users has led to general improvements in the code, both in terms of locating and correcting problem areas, as well as in adding and improving code options to meet the needs of users. Examples of overall corrections and improvements include,

1) Corrected $\mathrm{Be}-9(\mathrm{n}, 2 \mathrm{n})$ neutron emission spectra, which were incorrect.

2) Correctied $Z$ cone Reflection.

3) Corrected addxyz input.

4) Added zone volume user input option.

5) Added the option with tally types 11 and 12 , to tally in zone neutron absorption, rather than everytime a neutron enters a zone.

6) Allowed type 11 and 12 for empty (no material assigned) zones.

7) Added improved reflected calculations. 


\section{Running Time}

The below table presents results obtained using a collection of TART benchmark problems. All problems were run on each computer. This table summarizes timing results for the older TARTND code, that only runs on CRAY computers, as well as TART2000 and TART95 on a variety of computers.

\begin{tabular}{|c|c|c|c|}
\hline Code & Computer & $\begin{array}{l}\text { Running } \\
\text { Time } \\
\text { (Seconds) }\end{array}$ & $\begin{array}{l}\text { Ratio to } \\
\text { TARTNP } \\
\text { CRAY-YMP }\end{array}$ \\
\hline $\begin{array}{l}\text { TARTNP } \\
\text { TARTNP }\end{array}$ & $\begin{array}{l}\text { CRAY - YMP } \\
\text { CRAY-J90 }\end{array}$ & $\begin{array}{l}5396 \\
7727\end{array}$ & $\begin{array}{l}1.0 \\
1.43\end{array}$ \\
\hline $\begin{array}{l}\text { TART2 } 200 \\
\text { TART2000 } \\
\text { TART2 } 000 \\
\text { TART2 } 000 \\
\text { TART2 } 000 \\
\text { TART2 } 000 \\
\text { TART2000 } \\
\text { TART2 } 000 \\
\text { TART2 } 000 \\
\text { TART2000 } \\
\text { TART2 } 000 \\
\text { TART2000 } \\
\text { TART2000 } \\
\text { TART2 } 000 \\
\text { TART2000 } \\
\text { TART2000 } \\
\text { TART2000 } \\
\text { TART2000 } \\
\text { TART2 } 000 \\
\text { TART2000 } \\
\text { TART2 } 000\end{array}$ & $\begin{array}{l}\text { IBM-PC Pentium III/1000 } \\
\text { IBM-PC Pentium III/500 } \\
\text { DEC-Alpha Model } 5 / 625 \\
\text { IBM-PC Pentium II/400 } \\
\text { IBM-PC Pentium II/333 } \\
\text { DEC-Alpha Model } 5 / 300 \\
\text { IBM-PC Pentium II/266 } \\
\text { IBM-PC Pentium Pro/200 } \\
\text { IBM-PC Lap Top/233 } \\
\text { Power-MAC } 7500 / 275 \\
\text { iMAC } \\
\text { HP-735/125 } \\
\text { SUN E3000/166 } \\
\text { IBM-PC LapTop/133 } \\
\text { CRAY-YMP } \\
\text { IBM-RISC RS-6000 } \\
\text { CRAY-J90 } \\
\text { Meiko CS-2/66 } \\
\text { SUN SparC-20 } \\
\text { Power-MAC } 7500 / 100 \\
\text { SGI R4000/100 }\end{array}$ & $\begin{array}{r}243 \\
500 \\
516 \\
579 \\
697 \\
712 \\
855 \\
1185 \\
1301 \\
1350 \\
1664 \\
1834 \\
2107 \\
2990 \\
4262 \\
5739 \\
6095 \\
6225 \\
6315 \\
6446 \\
6953\end{array}$ & $\begin{array}{l}0.045 \\
0.09 \\
0.10 \\
0.11 \\
0.13 \\
0.13 \\
0.16 \\
0.22 \\
0.24 \\
0.25 \\
0.31 \\
0.34 \\
0.39 \\
0.58 \\
0.79 \\
1.06 \\
1.13 \\
1.15 \\
1.17 \\
1.21 \\
1.29\end{array}$ \\
\hline $\begin{array}{l}\text { TART95 } \\
\text { TART95 } \\
\text { TART95 } \\
\text { TART95 } \\
\text { TART95 } \\
\text { TART95 } \\
\text { TART95 } \\
\text { TART95 }\end{array}$ & $\begin{array}{l}\text { CRAY-YMP } \\
\text { HP-350 } \\
\text { DEC-Alpha } \\
\text { SUN } \\
\text { Meiko } \\
\text { SGI } \\
\text { IBM-RSIC } \\
\text { IBM-PC } 486 \mathrm{DX} 2 / 66\end{array}$ & $\begin{array}{l}4912 \\
4322 \\
6130 \\
9673 \\
9993 \\
10157 \\
14838 \\
18437\end{array}$ & $\begin{array}{l}0.91 \\
0.80 \\
1.14 \\
1.79 \\
1.85 \\
1.88 \\
2.75 \\
3.41\end{array}$ \\
\hline
\end{tabular}

When we compare the three codes all run on the same CRAY-YMP, we find that compared to the older TARTND code, TART95 was about $9 \%$ faster, and TART2000 is about $21 \%$ faster. So that not only has TART2000 been extended for more general uses, these extensions were accomplished with no lose in running time efficiency, i.e., TART2000 is actually faster than TART95.

You should also note the advantage of TART95 and TART2000 over the older TARTND in terms of their ability to be used on virtually any computer. For example, even a Laptop computer runs TART2000 over four times as fast as TARTND on a CRAY-YMP, and on a basically $\$ 3,000$ IBM-PC Pentium-III, $1000 \mathrm{MHz}$, TART2000 runs over twenty two (22) times faster than TARTND does on a multi-million dollar CRAY-YMP. 


\section{Why is Monte Carlo Used so much Today?}

The last point to note from these comparisons is how far we have come in terms of available inexpensive computer power in the three years between the release of TART95 and TART2000. When TART95 was released the fastest IBM-PC then available took 18,437 seconds to run this collection of theoretical problems. Even then we could foresee the potential of an inexpensive computer being able to run these problems in only about 3.4 as much time as it took on a CRAY-YMP. But I don't think anyone could foresee that just five years later we now have available IBM-PCs that can run this collection of problems in only 243 seconds; over twenty two (22) times faster than a CRAY-YMP. Compared to the PCs of only five years ago, not only does today's PC run these problems almost 76 times faster, but it does it at about half the cost.

Think about what a difference in running time of a factor of 76 means. A major expense of any scientific project is your salary, so time is money and it can be expensive - or inexpensive, depending on how you spend it. Consider that only five years ago if it took an entire 9 to 5,8 hour working day, to run a TART problem on an IBM-PC, today it would take less than 7 minutes to run the same problem, i.e., a factor of 76 faster. It should be noted, that this tremendous increase in available inexpensive computer power is one of the reasons that the use of Monte Carlo has expanded so much in recent years. Problems that we thought too time consuming to be practical just a few years ago, have now become routine.

\section{Why is TART so FAST?}

Some users make the mistake of assuming that since TART is so much faster than other codes that perform the same types of calculations, the results based on other codes must be better than those based on TART. When you use TART you will find that its results are just as accurate as those of other codes. So why is TART so fast?

There isn't any big secret to TART's speed: TART includes the three most important things necessary for generally efficient and accurate programming:

\section{EXPERIENCE! EXPERIENCE! EXPERIENCE!}

It is as simple as that. TART is based on over 30 years of continuous use and improvement. During this time roughly 80 work years of physicist/programmer time, and hundreds of work years of user experience, where incorporated into the code that we have today. To illustrate why TART is so much faster and still as accurate as other codes, I'll mention just a few points.

First is the use of multi-group data, including the multi-band method to account for self-shielding [1,7], as used by TART, compared to continuous energy cross sections used to other codes. Results using continuous energy cross sections have to be better, right? Not always! This is only true if you run a calculation for extremely long times so that you accurately sample ALL of the continuous energy cross sections. This is almost never done, and I know of no code that explicitly includes an estimate of the uncertainty in its results based on the enormous variation in continuous energy cross sections. In comparison, TART's approach is designed for the real world, and incorporates not only the best nuclear and atomic data, but also the best nuclear and atomic engineering.

For example, if we look at the U-238 cross sections we see capture cross sections that vary by roughly four orders of magnitude, and we can see that it is composed of very narrow resonances with relatively 
large energy intervals between resonances, i.e., the ratio of resonance spacing to width is about 100 to 1 . This data is VERY DIFFICULT to sample on a continuous energy basis. Indeed if you try it you will find that in order to obtain even a fairly accurate estimate of the average cross sections and distance to collision you would have to sample billions of histories. I don't know of any code that uses continuous energy cross sections that actually does this. They simply supply you with the "best" possible cross sections and assume that this will solve your problems. TART takes it a step further: not only does TART use the "best" cross sections, but also uses the "best" nuclear engineering. Again, consider the U-238 cross sections. Anyone who has taken a course in reactor physics knows that in this case the neutron flux will self-shield and we know the form of the self-shielding. Therefore we do not need all of the nittygritty details of each and every narrow capture resonance in order to perform an accurate transport calculation. Think about it: people have been successfully designing nuclear reactors for over 50 years, and yet only fairly recently have detailed cross sections become available. So how did people design their reactors? They did what TART now does: combine the "best" currently available nuclear data with the "best" nuclear reactor theory. In the case of TART the use of the multi-band method to account for resonance self-shielding $[1,7]$ allows it to use multi-group, rather than continuous energy cross sections, resulting in rapid convergence of calculations, compared to code that use continuous energy cross sections and take forever to converge. Most important for users to understand is that this is done with virtually no lose in accuracy in the TART calculations, indeed it is fair to say that since for reasonable running times the TART results converge and those of other codes do not, from the pragmatic viewpoint of obtaining accurate answers in a reasonable amount of time, the TART results are better.

I should also mention the unresolved resonance region, where by definition we do not know the cross sections on a continuous energy basis, but it can be accurately treated by the multi-band method used by TART. Ask yourself: what do codes that claim to use continuous energy cross sections do in the unresolved resonance region?

A second example of why TART is so fast is its treatment of geometry. Compared to other codes TART uses a very strict geometry, which places an additional burden on the user in terms of input preparation. But the pay off is that the input is easier to check and correct (using TARTCHEK) to improve reliability, and when the code starts to run it FLYS!!!

For example, TART insists that the users define every space point to be within a spatial zone. Other codes do not insist on this, so why does TART? The first reason is that without insisting on this it is not possible to check the input parameters for errors; checking is now simple and straightforward using TARTCHEK, and greatly improves the reliability of the input. Next, when TART runs it greatly accelerates tracking. How can a few holes in the geometry make such a big difference? Consider a simple problem involving 1000 spatial zones with each zone bounded by 6 surfaces. When a particle enters a spatial region that is not defined in the problem, i.e., is a "hole", the code has to track (ray trace) to the nearest bounding surface to determine what zone it will next enter. In this example it has to ray trace to the 6 bounding surfaces of each of the 1,000 spatial zones, to determine which of these surfaces is closest to the particle in its direction of travel, i.e., it has to ray trace to 6,000 surfaces. In contrast, with TART where a particle is always within a defined zone, in this example, we are inside one of the zones and we have to track (ray trace) to the nearest boundary of the zone. This only involving tracking to each of the bounding surfaces of this one zone, i.e., ray trace to 6 , rather than 6,000 surfaces. No wonder TART geometry is so much faster to track through. How much of an effect does this really make? TART and TARTCHEK use exactly the same geometry package. In the original method used by TARTCHEK to display 3-D objects, TARTCHEK used a general ray tracing technique that did not take advantage of TART geometry. When TARTCHEK was updated to take advantage of TART geometry the ray tracing to display 3-D objects ran up to 200 TIMES FASTER - not $200 \%$ - 200 TIMES!!! Pictures that took 
hours or all night to produce could suddenly be done in minutes or seconds. The difference was dramatic. You can see for yourself; use TARTCHEK to display 3-D views of your geometry and you will be amazed at how fast it can do it - and remember in ding it, it is using EXACTLY the same routines that TART uses to track through 3-D geometry. No wonder TART is so fast.

These are but a few examples of why TART runs so much faster than other codes, with essentially no lose in accuracy. Try it for yourself and see what you think.

As related to reliability, I'll also mention in passing the danger of using the default of other codes, that assume that whatever volume you have not explicitly defined is vacuum that the code can freely transport through. My experience has been that when a problem has an undefined volume in it, well over $90 \%$ of the time it is because it is an error. Other codes sweep this under the rug and make it appear that nothing is wrong, usually resulting in the wrong answer. In contrast TARTCHEK and TART2000 will quickly find these volumes and ask you to explicitly define them. This approach greatly improves the reliability of the TART input. 


\section{What Code should you be using?}

TART2000 completely supersedes all older versions of TART, and it is strongly recommended that users only use the most recent version of TART2000 and its data files. How do you know if you have the most recent version of the code and its data files? As soon as the code starts to run it identifies the version you are running and the dates of its data files. Below is the beginning of the code output report. Note, the code version: TART2000-1, Nov. '00, and the date of the four data files is 11/22/00. Note, also the newer 566 groups for the neutron data and 701 points for the photon data. If you are using an older version of the code or its data files, it is strongly recommended that you obtain the most up-to-date code and data; see, the below section on Availability.

TART2000 - Neutron-Photon Monte Carlo Transport (TART2000-1, Nov. '00)

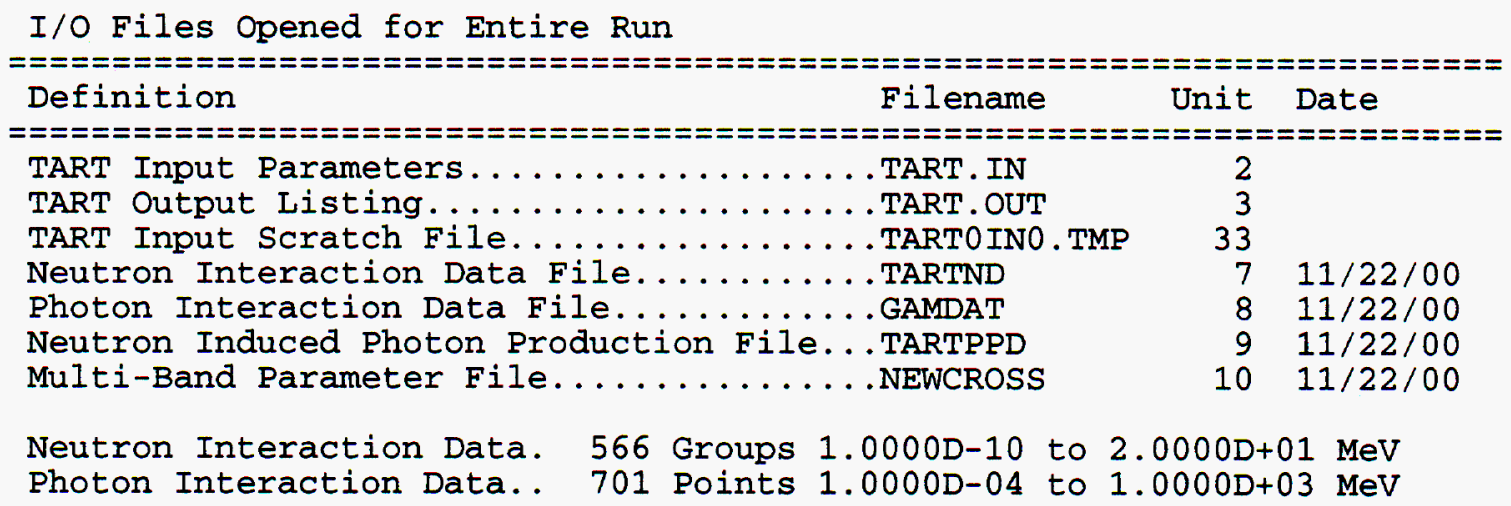

\section{Utility Codes}

In addition to the TART2000 code you should also be aware of the utility codes distributed with TART2000; of particular note are TARTCHEK and TARTAID. One of the most difficult tasks that you will face in using any 3-D combinatorial Monte Carlo code is to correctly define input parameters for the code, particularly to correctly define geometry. This is what TARTCHEK is designed to help you with. It is an interactive graphics code that will allow you to view and check your input parameters before you run TART2000. Even we so called "experts" on TART find that using TARTCHEK can greatly reduce the amount of time that we have to spend on input preparation, and even what is more important, greatly improve the reliability of our input parameters. In addition the TART2000 CD system includes a new code: TARTAID, which will allow you to interactively create TART input decks from scratch.

TARTCHEK can also help you analyze results by overlaying flux or energy deposition on your geometry. Instead of spending days or weeks wading your way through a thick output listing trying to understand the results, using TARTCHEK a few minutes after you finish a TART2000 calculation you can "see" the results overlaid on your geometry. Not only will this save you time, it can improve your overall understanding of the results, by showing you the "big picture" of how flux, deposition. etc., in each zone is related to that in all other zones. This is something that is very difficult to "see" regardless of how long you stare at an output listing. If you are not using TARTCHEK you are only making your job more difficult, and you don't know what you are missing.

TARTAID is another code you should be aware of. In addition to TARTCHEK, which can be used to 
check existing TART input, and display TART results, the TART2000 CD system also includes TARTAID. This code is designed to help you create TART input from scratch. It is particularly helping to define very detailed geometry, involving many spatial zones. For example to create a TART input deck involving 10,000 or even 100,000 spatial zones, takes only minutes using TARTAID.

You should also be away of the new utility codes MULTIPRO and TARTSUM, which will allow you to easily run many TART problems simultaneously, and then add together results from any number of TART problems, and produce a combined output file in EXACTLY the same format as any other single TART problem output file. Our computers are getting faster and faster, but we are running into the speed of light problem, where we can only get so much work done using a single processor. TART2000's approach to multiprocessing allows us to avoid this limit, in the sense that we can now compress the work that used to take many days, into a single day. This is true on either multiprocessing computers or a group of single processors computers. Just run your problems on ANY computer(s), using as many processors as you have access to, and TARTSUM will combine the results for you. Note, since the combined output file produced by TARTSUM is in EXACTLY the same format as any other single TART problem output file, if you are one of the many TART users who have utility codes to further process TART output results - not to worry - your utility codes will work on the combined file, exactly the same way they work on the results of a single TART run.

\section{Documentation}

Although TART2000, supersedes all earlier versions of TART, the most complete documentation for TART is still,

TART95: A Coupled Neutron-Photon Monte Carlo Transport Code, Lawrence Livermore National Laboratory, UCRL-MA-121319, July 4, 1995, by D. E. Cullen, A.L. Edwards and E.F. Plechaty

This document, as well as all other TART documentation, is now available on-line at the TART website, htp://reddog1.1lnl.gov 


\section{Availability}

At Livermore, for copies of the system, contact me. Outside of Livermore, contact your local computer code center - within the United States, the Radiation Safety Information Computational Center (RSICC), Oak Ridge National Laboratory (e. mail: jib@ornl.gov), outside of the United States, the OECD Nuclear Energy Agency/Data Bank (NEA/DB), Paris, France (e. mail: sartori@nea.fr).

\section{Code Installation}

The code is distributed with detailed instructions concerning installation and testing of the code. These instructions are periodically updated for distribution with the code, to insure that the instructions are as up-to-date as possible, and exactly correspond to the version of the code that you will be implementing and using. As such, installation instructions will not be included here. 


\section{References}

[1] "TART95: A Coupled Neutron-Photon Monte Carlo Transport Code," Lawrence Livermore National Laboratory, UCRL-MA-121319, July 1995, by D.E. Cullen, A.L. Edwards and E.F. Plechaty

[2] "TART96: A Coupled Neutron-Photon 3-D, Combinatorial Geometry Monte Carlo Transport Code," Lawrence Livermore National Laboratory, UCRL-ID-126455, November, 1996, by D.E. Cullen.

[3] "The 1996 ENDF/B Pre-Processing Codes," The International Atomic Energy Agency, Vienna, Austria, IAEA-NDS-39, Rev. 9, November 1996, by D.E. Cullen.

[4] "THERMAL: A Routine Designed to Calculate Neutron Thermal Scattering," Lawrence Livermore Nation $\varepsilon$ Laboratory, UCRL-ID-120560-Rev-1, Sept. 1995, by D.E. Cullen.

[5] “A Temperature Dependent ENDF/B-VI, Release 4 Cross Section Library," Lawrence Livermore National Laboratory, UCRL-ID-127776, by D.E. Cullen.

[6] "EPDL97: the Evaluated Photon Data Library, '97 Version," Lawrence Livermore National Laboratory, UCRL--50400, Vol. 6, Rev. 5, by D.E. Cullen.

[7] "Nuclear Cross Section Preparation", by D.E. Cullen, Chapter 1, Volume I, "Handbook of Nuclear Reactions Calculations," editor Yigal Ronon, CRC Press, Boca Raton, Florida (1986).

[8] "TART97: A Coupled Neutron-Photon 3-D, Combinatorial Geometry Monte Carlo Transport Code," Lawrence Livermore National Laboratory, UCRL-ID-126455, Rev. 1, November, 1997, by D.E. Cullen, and "TART98: A Coupled Neutron-Photon 3-D, Combinatorial Geometry Time Dependent Monte Carlo Transport Code," Lawrence Livermore National Laboratory, UCRL-ID-126455, Rev. 2, November, 1998, by D.E. Cullen

[9] "A Simple Model of Photon Transport", by D.E. Cullen, Nuclear Instrumentation and Methods in Physics Research B101 (1995) pp 499-510. The original extended form of this paper is now available online at the TART website, http://reddog1.llnl.gov

[10] "A Temperature Dependent ENDF/B-VI, Release 7 Cross Section Library," Lawrence Livermore National Laboratory, UCRL-ID-127776, Rev. 1 (2000), by D.E. Cullen. 


\section{Appendix: Summary of New Conventions and Options}

To help explain and illustrate the use of the new options the TART2000 CD distribution includes example input decks. I encourage you to use TARTCHEK to look at these examples - particularly using 3-D views, so you can see them better.

\section{Biggest Changes for TART2000 vs. TART98}

\section{Add section here}

\section{Biggest Changes for TART98 vs. TART97}

In terms of physics, the biggest change is in the treatment of photons. Compared to TART97, TART98 includes a much more detailed representation of photon cross sections, and a greatly improved treatment of coherent and incoherent scattering. TART98 also includes expanded tally and output options for photon results.

TARTAID is available for the first time with TART98 CD. This has already become a very popular code. I designed TARTAID as somewhat of a complement to TARTCHEK, to deal with the problem of preparing and checking TART input before it is used in actual TART calculations. What I didn't foresee is that if you are using TARTAID many of the classic errors that TARTCHEK checks for cannot occur, and rather than complementing TARTCHEK, TARTAID is somewhat replacing it.

The other important change is EXPERIENCE!!! Again, I cannot stress how important this is for any code. In the case of TART each successive version of the code includes the operating experience of the many people who are now using the code. With each passing version of TART reliability and accuracy are improved, mostly based on feedback from users - such as yourself.

\section{Biggest Changes for TART97 vs. TART96}

There is no limit on input parameters. You can have any number of surfaces, zones, bounding surfaces, materials, sources, e.g., you can have a million zones, with a different material in every zone, if you want to do burnup calculations.

This also means there is no lower limit. Earlier versions of TART were dimensioned to handle large problems. Because of this the code would start at about $50 \mathrm{MB}$ and then decrease in size. This caused startup problems on smaller computers. TART97 starts at about $1 \mathrm{MB}$ and increases to meet the needs of your specific problem; most problems will only use 3-4 MB.

Any input line can now be continued onto any number of continuation lines. With earlier versions of TART some input, particularly complicated sources, could not be continued from one line to another, which made input preparation difficult. You will find that being able to continue any input line, it is much easier to prepare input. Some of the following new options, such as cloning, rotation and spatial translation, were recommended by TART users, and are also designed to simplify preparation of TART input. If you have ideas to even further simplify input preparation, I'd love to hear them.

\section{The new input Options}




\section{Cubic}

xcubic nb x0 y0 z0 d c b a ycubic nb $x 0$ y0 z0 d c b a zcubic nb x0 y0 z0 d c b a

a cubic, rotationally symmetric about an axis - the equations are,

xcubic: $(y-y 0)^{2}+(z-z 0)^{2}=R(x)^{2}$

$$
=a(x-x 0)^{3}+b(x-x 0)^{2}+c(x-x 0)+d
$$

ycubic: $(\mathrm{x}-\mathrm{x} 0)^{2}+(\mathrm{z}-\mathrm{z} 0)^{2}=\mathrm{R}(\mathrm{y})^{2}$

$$
=a(y-y 0)^{3}+b(y-y 0)^{2}+c(y-y 0)+d
$$

zcubic: $(x-x 0)^{2}+(y-y 0)^{2}=R(z)^{2}$

$$
=a(z-z 0)^{3}+b(z-z 0)^{2}+c(z-z 0)+d
$$

nb - Surface Number

$\mathrm{x} 0, \mathrm{y} 0, \mathrm{z} 0 \quad$ - Center coordinates

$\mathrm{d}, \mathrm{c}, \mathrm{b}, \mathrm{a} \quad$ - Coordinates of the cubic

The radius along one axis is represented as a cubic. By defining zones using a cubic and planes perpendicular to the axis of the cubic, you can reproduce almost any surface, using different cubic parameters to apply along different intervals of the axis; exactly as we think in terms of performing cubic spline fits.

You can reproduce almost any surface depending on a, b, c and d - spheres, ellipses, cylinders, cones, parabola, hyperbola, plus more complicated shapes.

WARNING it is $\mathrm{R}^{2}$, NOT $\mathrm{R}$, that is represented by a cubic.

Example problem: NEWCUBIC.IN

\section{Torus}

xtorus nb x0 y0 z0 a b c ytorus $\mathrm{nb} \times 0 \mathrm{y0} \mathrm{z0}$ a b c ztorus $\mathrm{nb} \times 0 \mathrm{y0} \mathrm{z0}$ a b c

a torus aligned with an axis - the equations are,

xtorus: $[(\mathrm{x}-\mathrm{x} 0) / \mathrm{a}]^{2}+[(\mathrm{r}-\mathrm{c}) / \mathrm{b}]^{2}=1$

$$
\mathrm{r}^{2}=(\mathrm{y}-\mathrm{y} 0)^{2}+(\mathrm{z}-\mathrm{z} 0)^{2}
$$

ytorus: $[(y-y 0) / a]^{2}+[(r-c) / b]^{2}=1$

$$
\mathrm{r}^{2}=(\mathrm{x}-\mathrm{x} 0)^{2}+(\mathrm{z}-\mathrm{z} 0)^{2}
$$

ztorus: $[(\mathrm{z}-\mathrm{z} 0) / \mathrm{a}]^{2}+[(\mathrm{r}-\mathrm{c}) / \mathrm{b}]^{2}=1$

$$
r^{2}=(x-x 0)^{2}+(y-y 0)^{2}
$$


$\mathrm{nb} \quad$ - Surface Number

$\mathrm{x} 0, \mathrm{y0}, \mathrm{z0} \quad-$ Center coordinates

$\mathrm{a}, \mathrm{b}, \mathrm{c}$ - Coordinates of the torus

If $\mathrm{a}=\mathrm{b}$, it is a circular torus, otherwise it is an elliptical torus.

Example problem: NEWTORUS.IN

Rotation about the $\mathrm{X}, \mathrm{Y}$ or $\mathrm{Z}$ axis

xrotate ang is1 thru is 2

xrotate ang is 1 is 2 is $3 . . . .$.

yrotate ang is1 thru is 2

yrotate ang is 1 is 2 is $3 . . . .$.

zrotate ang is1 thru is 2

zrotate ang is 1 is 2 is3......

A rotation of surface(s) about an axis by a clockwise angle ang (degrees) looking up the axis. Rotation is about the ORIGIN - not the center of the surface. Note, this differs from surfp and srotate input, which can only be used to rotate surfr input about the center of the surface.

ang - angle of rotation in degrees

is 1 thru is 2 - rotate surface numbers is 1 thru is 2

is 1 is 2 is $3 \ldots$ - rotate the listed surface numbers

Surfaces can be rotated one or more times, and the rotation is cumulative and order dependent.

Any linear or quadratic surface may be rotated. Cubic and torus MAY NOT be rotated (at least yet).

WARNING - these options are executed immediately when they are read from a TART input deck. Therefore all surfaces to be rotated MUST be defined before they can be rotated, and the order of rotations is important.

WARNING - for TARTCHEK users, the lower, left hand plot, is looking at the front of your geometry in the $(z, x)$ plane, looking UP THE Y AXIS. The upper, left hand plot, is looking down at the top of your geometry in the $(\mathrm{z}, \mathrm{y})$ plane, looking DOWN THE X AXIS. The lower, right hand plot, is looking at the right hand size of your geometry in the $(y, x)$ plane, looking DOWN THE $Z$ AXIS. As a result, a clockwise rotation about the $\mathrm{y}$ axis will appear clockwise in the lower, left hand plot. However, a clockwise rotation about the $\mathrm{x}$ axis will appear COUNTERCLOCKWISE in the upper, left hand plot, and a clockwise rotation about the $\mathrm{z}$ axis will appear COUNTERCLOCKWISE in the lower, right hand plot. This isn't an error - it is merely a result of your perspective when viewing TARTCHEK displays.

Example problems: NEWHEX.IN, NEWROT.IN (1 rotation) and NEWROT2.IN (2 rotations)

\section{Translation of Spatial Coordinates}

addxyz xadd yadd zadd is1 thru is 2

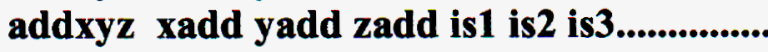


Add $(x, y, z)$ to the current center of surfaces.

xadd, yadd, zadd - add to the current $(\mathrm{x} 0, \mathrm{y} 0, \mathrm{z} 0)$ center coordinates of surfaces

is 1 thru is 2 - add to surface numbers is 1 thru is 2

is 1 is 2 is $3 \ldots \quad$ - add to the listed surface numbers

Any surface may be translated, any number of times - and the results are cumulative.

This can be used to translate an entire object or objects to a new location, by translating all bounding surfaces by the same amount. It also simplifies input by allowing you to ignore the final position of a collection of surfaces, and input them as if they are at the origin - then later "add" their final center coordinates.

WARNING - these options are executed immediately when they are read from a TART input deck. Therefore all surfaces to be spatially translated MUST be defined before they can be spatially translated.

Try: Adding this to any of the example input

Cloning (Duplicating) Surfaces

\section{clones $n s$ is1 thru is 2}

clones ns is1 is 2 is3.....

Clone (copy) a surface any number of times. Surface ns is copied to define surfaces is 1 thru is 2 , or is 1 is 2 is $3 \ldots . .$.

ns - surface number to clone (MUST be defined)

is1 thru is 2 - make surface numbers is1 thru is2 identical to surface ns

is 1 is 2 is 3 .. - make the list of surface numbers identical to surface ns

Limitations: surface number ns MUST be defined BEFORE it can be cloned (copied). The surface numbers is 1 thru is 2 or is 1 is 2 is $3 . .$. MUST NOT be defined.

Any surface may be cloned, any number of times.

This option can be used to minimize input preparation when you have a number of identical surfaces that will finally be located at different locations. You can input a surface once, clone it, and then later translate and/or rotate the clones to their final locations.

WARNING - these options are executed immediately when they are read from a TART input deck. Therefore the surface to be cloned (ns) MUST be defined before it can be cloned.

Example Problems: NEWHEX.IN, NEWROT.IN and NEWROT2.IN

Reduced, Reflecting Geometry

xabove $x 0$

yabove y0 
zabove $\mathbf{z 0}$

xbelow x0

ybelow y0

zbelow z0

For users who only want to model $1 / 2,1 / 4$ or $1 / 8$ of symmetric geometry, these options can be used to: 1) define additional $\mathrm{x}, \mathrm{y}$ and/or $\mathrm{z}$ planes, 2) add these planes as boundaries of ALL zones, 3) add additional, reflecting zones on the "other" side of the planes.

$\mathrm{x} 0, \mathrm{y} 0, \mathrm{z} 0 \quad-$ a plane perpendicular to the axis is defined at one of these coordinates.

"above" means transport above this plane - the reflecting zone is below this plane.

"below" means transport below this plane - the reflecting zone is above this plane.

With earlier versions of TART in order to accomplish this you had to include the surface of the reflecting zone explicitly as a bounding surface of every zone. With this new input option this is automatically done for you.

For TARTCHEK users, to see the effect of inserting these planes, use the above/below options on the "Surface" page.

WARNING: These planes are inserted into the geometry AFTER ALL input has been read - they CANNOT be rotated or transformed in ANY way. It is suggested that as a reminder to yourself, you always locate these options at the end of your TART input deck after all other geometric input parameters have been defined.

Try: Adding this to any of the example input 


\section{New Sources}

These sources can be used to sample sources from irregularly shaped zones. Unlike the other sources, these sources reject a sample if it is not inside a zone number in the range $\mathrm{nz} 1$ through $\mathrm{nz} 2$. These three new sources are for a sphere, cylinder, or rectangular box. For sampling select whichever of these shapes corresponds "best" to the shape of your actual zone numbers nzl through $\mathrm{nz} 2$.

\section{RESTRICTIONS}

1) nzl MUST be less than or equal to $n z 2$.

2) If none of 10,000 consecutive samples from the defined volume is within zone numbers nz1 through $\mathrm{nz2}$, it is assumed you made a mistake and the code will terminate. This prevents the code from going into an infinite loop of sampling and rejecting forever.

\section{source19 nz1 thru $\mathrm{nz2}$ ri ro $[\mathrm{x0} \mathrm{y0} \mathrm{z0}]$ \\ s19 nz1 thru nz2 ri ro [x0 y0 z0] \\ s19g nz1 thru nz2 ri ro [x0 y0 z0]}

A spherical shell source of inner radius ri, and outer radius ro, centered at x0, y0, z0. Use source19 or s19 for neutrons, and $\mathrm{s} 19 \mathrm{~g}$ for photons.
nz1 - lowest zone number to sample from
nz2 - highest zone number to sample from
ri - inner radius of sphere
ro - outer radius of sphere
$\mathrm{x} 0, \mathrm{y} 0, \mathrm{z} 0 \quad$ - center of the sphere (optional, defaults to $0,0,0$ )

source20 nz1 thru nz2 z1 z2 ri r0 [x0 y0]

s20 nz1 thru nz2 z1 z2 ri r0 [x0 y0]

s20g nz1 thru nz2 z1 z2 ri r0 [x0 y0]

A cylindrical shell source, aligned with the $\mathrm{z}$ axis, extending along the $\mathrm{z}$ axis from $\mathrm{z} 1$ to $\mathrm{z} 2$, of inner radius ri, and outer radius ro, centered at $\mathrm{x} 0$, y0. Use source 20 or $\mathrm{s} 20$ for neutrons, and $\mathrm{s} 20 \mathrm{~g}$ for photons.

$\begin{array}{ll}\mathrm{nz} 1 & \text { - lowest zone number to sample from } \\ \mathrm{nz} 2 & \text { - highest zone number to sample from } \\ \mathrm{z} 1 & \text { - lower } \mathrm{z} \text { limit of cylinder } \\ \mathrm{z} 2 & \text { - upper } \mathrm{z} \text { limit of cylinder } \\ \mathrm{ri} & \text { - inner radius of cylinder } \\ \text { ro } & \text { - outer radius of cylinder } \\ \mathrm{x} 0, \mathrm{y} 0 & \text { - center of the sphere (optional, defaults to } 0,0 \text { ) }\end{array}$

Note, for a cylinder aligned with an axis other than the $z$ axis, use sentl 30 (neutrons) or sentl 43 (photons) to rotate the coordinates. 


\section{source21 nz1 thru nz2 x1 x2 y1 y2 z1 z2}

s21 nz1 thru nz2 x1 x2 y1 y2 z1 z2

s21g nz1 thru nz2 x1 x2 y1 y2 z1 z2

A rectangular box in $(\mathrm{x}, \mathrm{y}, \mathrm{z})$, extending in $\mathrm{x}$ from $\mathrm{x} 1$ to $\mathrm{x} 2$, in $\mathrm{y}$ from $\mathrm{y} 1$ to $\mathrm{y} 2$, and in $\mathrm{z}$ from $\mathrm{z} 1$ to $\mathrm{z} 2$. Use source 21 or $\mathrm{s} 21$ for neutrons, and $\mathrm{s} 21 \mathrm{~g}$ for photons.

nz1 - lowest zone number to sample from

$\mathrm{nz2} \quad$ - highest zone number to sample from

$\mathrm{x} 1-$ - lower $\mathrm{x}$ limit of box

$\mathrm{x} 2 \quad-$ upper $\mathrm{x}$ limit of box

y1 - lower y limit of box

y2 - upper y limit of box

z1 - lower $\mathrm{z}$ limit of box

z2 - upper $\mathrm{z}$ limit of box

There are no examples of these sources included here.

\section{Defining Zone Volume by User Input}

volume nz1 vz1 nz2 vz2 nz3 vz3.....

The volume (in cc) for zones $\mathbf{n z 1}, \mathbf{n z 2}, \mathbf{n z 3} \ldots$ is defined by input to be vz1, vz2, vz3.., respectively. TART will attempt to analytically calculate the volume of each zone. When it cannot analytically calculate the volume you can use the input option mevdisk or mevplane to statistically sample the volume. In order to accurately statistically sample volumes can be very time consuming. This input option allows you to statistically sample volumes only once, and then in all following calculations you need merely define your zone volumes by input. This will allow you to correctly define volumes that can effect special output, such as deposition in MeV/cc or MeV/grams, see probm and sentl 35 .

Example Input: The following input line defines the volume of zones 217 and 23 to be 1.34, 13.7 and $213.6 \mathrm{cc}$, respectively,

\section{Volume $2 \quad 1.34 \quad 17 \quad 13.7 \quad 23 \quad 213.6$}

Restrictions: The zone numbers nz1, nz2, nz3... MUST be 1 to the maximum number of zones used in the problem, and all volumes be vz1, vz2, vz3... MUST be positive. The volumes that you input are used by TART without questioning their accuracy, and no attempt is made to analytically calculate the volumes of these zones.

\section{Changes in sentinels}

Photon tally bin sentinel

sentl $20(0)$

This sentinel was not used in earlier version of TART. 
Starting with TART98 this sentinel can be used to define the Photon tally bin sentinel. If 0 , the default, 70 photon tally bins will be used. If 1 , there will be 175 photon tally bins. If 2 , there will be a full 700 photon tally bins. For neutrons, see sentl 46.

Do not limit the energy range of transport and scoring

\section{sentl 8 and 9}

These sentinels define the minimum neutron (sentl 8) and photon (sentl 9) energy below which particles cannot transport.

DO NOT use these, unless you really want to limit the minimum energy of neutrons and photons. TART will now use the minimum energy of the data read from the data files - which for neutrons differs for 175 and 650 groups.

\section{sentl 13 and 14}

These sentinels define the minimum neutron (sentl 13) and photon (sentl 14) energy below which particles cannot tally (contribute to output results).

Similar to sentl 8 and 9 above - DO NOT use these, unless you really want to limit the minimum editing energy of neutrons and photons.

\section{sentl 15 and 16}

These sentinels define the maximum neutron (sentl 13) and photon (sentl 14) energy above which particles cannot tally (contribute to output results).

Similar to sentl 8 and 9 above - DO NOT use these, unless you really want to limit the maximum editing energy of neutrons and photons. Note, soon TART will be extended to higher energies, so get used to not using these options now.

\section{New random number sequence selection}

\section{sentl 12}

The code now has 2,510 sequences, one trillion (10 12 ) samples apart. Input 0 (the default) to 2509 will use the selected sequence. Any other input is a fatal ERROR.

WARNING - this replaces the earlier definition of this sentinel, where the random number seed was entered; seen. TART95 documentation.

\section{Highly Recommended Options}

For compatibility with earlier versions of TART, by default the following options are turned off, unless the user specifies by input that they be turned on. It is Highly Recommended that you turn on ALL of the following options. 


\section{sentl 20}

For neutron problems turn on resonance self-shielding. This can make problems run 20 to $30 \%$ longer, but without accounting for self-shielding the results can be completely unreliable.

\section{sentl 25}

For photon problems turn on fluorescence. If no photons get down to low energies, this will have no effect on running time. However, if they do, this option is REQUIRED to obtain reliable answers.

\section{sentl 39}

For neutron problems turn on thermal scattering. If no neutrons get down to thermal energies, this will have no effect on running time. However, if they do, this option is REQUIRED to obtain reliable answers.

\section{sentl 54 (0)}

The definition of the number of neutrons per fission. If this option is 0 (the default), the average number of neutrons per fission is used. If this option is 1 , the energy distribution of the number of neutrons per fission is sampled; this later option is designed for use in reactor noise analysis calculations.

Restrictions: This option can only be used for neutron source problems; it cannot be used for criticality calculations. If you try to use this option for criticality calculations the code will abort.

\section{sentl 55 (0)}

Spontaneous fission source. If this option is 0 (the default) all source neutrons are treated as uncorrelated, as far as time and position of emission, and you may specify the neutron source energy spectrum by input. If this option is positive (see, restrictions below), the neutron source is treated as a spontaneous fission source, with the emission of neutrons correlated, as far as time and position of emission. The total source strength for any problem is still defined by the product of sentinels 2 and 3, however when this option is used, the number of neutrons per fission is used to emit $v(E)$ successive neutrons at the same time and position; the neutron energies and directions are sampled independently from the appropriate distributions.

Example input: sentl 55 98252, will cause the neutron source to be treated as a Cf252 spontaneous fission spectrum.

Restrictions: This option can only be used for neutron source problems; it cannot be used for criticality calculations. If you try to use this option for criticality calculations the code will abort. Currently this option is limited to ZA = 94240 (Pu240), 96242 (Cm242), $96244(\mathrm{Cm} 244)$, and 98252 (Cf252). When you use this option, you MUST use a neutron fission spectrum source (sentl 4 default), and the angular distribution MUST be isotropic (sentl 6 and 7 defaults).

\section{sentl $56(0)$}

Tally 11 and 12 in zone absorption. Normally when using tally type 11 or 12 for neutrons, every time a neutron enters the zone its coordinates are tallied in a binary file. When this option is 1 , neutron 
coordinates are only tallied when a neutron is absorbed in the zone. This option can be used to better simulate neutrons detector response, where the response is due to neutron ansorption.

Restrictions: this option should not be used for void (empty) zones, otherwise you will not obtain any tally. 